\title{
BIM-BASED ANALYSIS OF SPATIAL PROPERTIES IN BUILDING LAYOUTS
}

\author{
HUGO SHEWARD \\ School of Architecture and Design, University of Kansas, USA
}

\begin{abstract}
We describe here methodologies for embedding geometric and topological operations in contemporary Building Information Modeling (BIM) Computer Aided Design (CAD) software, along with the computational implementation of domain specific evaluations; this is done to achieve the integration between spatial-based spatial properties evaluations and architectural design workflows. This research describes methodologies and computational technologies used for the development of a computational design assistant in the context of laboratory buildings in Preliminary Concept Design (PCD).

Keywords: BIM, morphology derivation, design assistances, concept design, building performance simulation
\end{abstract}

\section{INTRODUCTION}

This paper describes research in the automation of derivation of computational building models' spatial properties for the purpose of ventilation systems engineering, such systems are an important design assessment parameter when performing design decisions and affect the performance of the building [1]. We develop computational technologies to allow computer software to derive spatial relationships that affect the environmental conditions inside laboratories. Ventilation systems engineering is intrinsically connected to the design of laboratories, different types of these are applied at almost every step of the design process from PCD to Design Development (DD).

Even though collaboration between a Architects and Engineers (AEs) is a common practice in later stages of design, this has not been the case for PCD, this is mainly the result of the speed in which design alternatives are produced during PCD and the complexity of traditional HVAC simulation tools, and the fact that most of these require complex data modeling, before any feedback can be provided to designers [2]. Design decisions taken during PCD can affect the performance of laboratory facilities and the ventilation system itself, most of the time these are made mainly by the architectural designer, and these are based on ventilation engineering rules of thumb. We explore how to automate and improve on traditional practices in the estimation of ventilation systems engineering during PCD, and how the engineering feedback involved in these practices can be produced by a domain specific Building Information Modeling (BIM) software. For this purpose, we developed a software; Laboratory Ventilation Design Assistance (LVDA) which is designed to be used by AE's when evaluating PCD of ventilation systems engineering of laboratories.

\section{THE IMPORTANCE OF VENTILATION SYSTEMS IN LABORATORIES}

Commonly known as ventilation driven facilities, laboratories demand high number of air exchanges and well-planned directional air flows [3]-[5], this becomes of extreme importance when dealing with high levels of Biosafety Level (BL) laboratory spaces. Also, they require $100 \%$ fresh air since their equipment exhaust cannot be recirculated, therefore more air needs to be brought into the building to make up for the exhaust. These requirements translate to higher energy consumption. Also, the design of the air distribution network in a laboratory is commonly considered an environmental safety measure. The directional airflow 
patterns in laboratory layouts are designed to be negative towards all laboratory spaces. Traditional practices in the design of air flow have been based on the pressure differential between spaces, this is accomplished by increasing the amount of air flow exhaust within the laboratory spaces [3], [6], thus increasing the energy consumption. Bennett et al. [6] pointed that the inward air flow can be more accurate for controlling the air flow patterns. The design of the inward directional airflows is directly connected to the layout morphology and the air pressurization of the different spaces in it. The spatial arrangement of the layout also affects the performance of the ventilation system regarding the required air pressure of the ventilation branches. The locations of served spaces within the layout and the typology of layout, impact in both the length and number of turns that the ventilation branches must have to reach all the required spaces.

\subsection{Domain of implementation}

The level of abstraction of a design might not be critical when dealing with paper-based representations, but it becomes relevant when the design representation is a computational model for supporting automation. Although there is not a widely adopted standard for the level of completeness of computational models in architecture and engineering (AE), recent efforts have tried to define these for BIM models [7], [8]. Among these the General Services Administration (GSA), Facilities Standards for the Public Building Service P100, establishes design standards for new buildings, among these; the levels of design development are defined as: Preliminary Concept Design (PCD), Late Concept Design (LCD), Concept Design (CD), and Design Development (DD). In the P100, the semantic content for each of these is defined making it suitable for the research developed here. In it PCD models are defined in terms of the content as follows: placement and massing of the building are defined; program spaces are identified only at a departmental level, circulation spaces both human and vehicular are identified, no internal partition walls or wall openings, basic definition of building boundary surfaces. Later stages of design, such as LCD, DD and CD follow.

Few objects usually are included in PCD BIM besides space objects; among these; building envelope, partitions among spaces are represented either with wall objects or virtual walls. These PCD BIM models are usually developed for the purpose of massing and spatial layout studies. In the case of laboratories, the main spatial referent for layout programming is the laboratory module. The sizing of these allows AE's to define the PCD layout of the building's structural grid, and to have an approximation to the expected occupancy loads of the facility. Based on the design requirements the laboratory module can include two other program components besides the laboratory space: office and lab support space. The spatial relations among these will affect both human circulation conditions and dimensioning of the building's ventilation systems engineering.

\subsection{PCD of laboratories and engineering assessment}

In the case of laboratory design, there is no precise framework for how engineering expertise is brought in to PCD, particularly how spatial properties play a role in the estimation of the ventilation system, most of the time engineers will get involved once the massing of the building and internal layout has been completed by the architect. Often the PCD tends to optimize the spatial adjacency of the facility, but in terms of ventilation engineering there is no optimization but the application of engineering rules of thumb regarding the floor to floor clearance required [9]. In traditional PCD of laboratories, after the model is completed by the architect, it is handed to the engineer who extracts its spatial properties, add to them his or 
her expertise in terms of code requirements and best practices to produce a ventilation engineering data model. Then after the cooling loads have been estimated the results are returned to the architect, who based on the results might explore other design alternatives. If new alternatives are explored, a new cycle of engineering estimation is conducted. The overall time for each of these iterations might be hours in the best of cases. It is easy to infer the reasons for the inefficiencies in this process; only in data exchanges/inputs, there are at least 9 steps, even more; some of these rely on manual extraction/manipulation of data. Therefore, making them prone to error.

\subsection{Building Performance Simulation (BPS) integration to laboratory design}

In general terms Ventilation system design using BPS's is performed after the spatial arrangement of the facility is consolidated and the material specification and configuration of the building envelope is well known by the design team. In traditional building design practices is during $\mathrm{CD}$ or FCD, when the HVAC engineer will conduct a detailed analysis of the heat loads the facility, define the ventilation rates, determine the air-flows, and propose the duct layout including the location of vertical drops as well as other pieces of equipment. The results of the CD or FCD HVAC engineering analysis can generate a set of design revisions in order to properly fit the HVAC components and their requirements [10], [11], which due to the state of completeness of the design can produce revisions and time delays. The previously described process is supported using BPS tools, many of these tools features makes it hard for any implementation of collaborative design environments in early stages of design [12].

Efforts have been made to automate areas of BPS to make them more suitable to early stages of design, still BPS requires for a certain level of semantic content in the model, such is the case of; material definition for walls, and sizing and placement of doors and windows. This content, usually available in CD or FCD is not commonly part of PCD semantics. Other reasons can also be pointed as to why BPS's are not suitable for PCD; the speed in which design changes happen during this phase [13], the time required to prepare and complete BPS, since once the analysis is completed the entire design might have changed making the results obsolete [2]. Both Holzer [13] and Chaszar [14] indicate that software's results might not enable interdisciplinary collaboration and that different domain semantics can create friction among AE teams. Holzer (2009) also points among the issues limiting collaboration, the need for team members to reflect in privacy regarding the proposed solutions.

\subsection{Current trends in engineering assistances}

The development of computational BPS tools has been going on for over 40 years. The range of these varies greatly from; excel based tools to highly advanced software. Trcka and Hensen [15] identify three generations of BPSs; the first; analytical formulations and simplified assumptions, the second; numerical methods, and partial integration of performance aspects of buildings, the current generation of BPS can capture reality better and are fully integrated regarding different performance aspects. In the area of HVAC there are roughly four categories of BPS's, these are based in the problem they are trying to analyse:

1. Equipment sizing: Carrier HAP, Trane Trace, Energy Plus, Design-Builder, MC4suite etc. 
2. Energy performance: Carrier HAP, Trane Trace, Energy Plus, DOE-2, Equest, ESP-R, IDA ICE, Trnsys, Hvacsim+, VA114, Simbad, Building Energy Analyzer, DesignBuilder, etc.

3. System optimization and controls: Genopt (generic), Contam, Energy Plus, ESP-R, Trnsys, Dymola, etc.

4. Duct sizing: AFT Fathom, Dolphin, Duct Calculator, Duct size, Pipe-Flo, Python, Indus, Cymap, etc.

Most of the previously listed have been developed for the purpose of HVAC engineering design; therefore, require the construction of domain specific data models, with properties, such as; transmittance values, operating schedules, equipment types, control strategies, and utility rates. Many of these have complex User Interfaces (UI), also the feedback produced might be hard to understand by experts [16]. Current efforts in BPS development concentrate on improving the integration of these to the overall building design process [17]. Three main areas being researched: the simplification of the calculations being performed [18], the simplification of the simulation data model being used and the automating generation of simulation models for the execution of BPS [19], [20]. This research takes on these trends and goes a step further in the effort of integration to design process by embedding engineering estimation within BIM software.

\section{METHODOLOGY FOR ACQUIRING VENTILATION SYSTEMS ENGINEERING}

We have conducted an extensive research dealing with widely recognized compilations of best practices and normative calculations applied to the engineering of ventilation systems in laboratory, these range from energy standards [21], design requirements [5], design guidelines [3], [4], [22]-[24], and HVAC engineering [25], [26]. From these we have extracted provisions dealing with issues regarding engineering of ventilation systems in the following areas:

1. Recommended design practices in terms of operational procedures

2. Code compliance for the design of ventilation systems

3. Minimum ventilation requirements for the operation of the facility

4. Best practices for the safety conditions for the facility

5. Systems serviceability provisions

\subsection{Capturing formulaic and domain expert data}

The methodologies for capturing expert data vary depending on the area of expertise being processed. For instance, in some areas of architecture it might be the size of service areas in a building regarding the usable square footage, in engineering it might be the types of connectivity that a pre-cast concrete beam needs when installed, or multiple forms of expertise which when combined represent complicated areas of design knowledge [27]. In the case of ventilation engineering for laboratories, the expertise is in most cases based on the relation between the organization of the layout, space usage and the requirements of spaces in terms of: environmental requirements, scientific processes, internal equipment or mechanical systems. In this research we capture the properties of the program components in the layout, and the instance properties that play a role in the derivation of spatial properties of it. For this purpose, we have developed a comprehensive space instance classification. This has been collected in a human readable file which includes the following space types: 
1. General chemistry

2. Radio chemistry

3. Research

4. Hospital or clinical

5. Biological containment

6. Animal research

7. Isolation/clean rooms

8. Materials testing

9. Electronics/instrumentation

10. Teaching

11. Laboratory Support

12. Offices

13. Toilet
14. Lockers/showers

15. Conference/ Break rooms

16. Corridor

17. Service Corridor

18. Elevators

19. Loading docks

20. Housekeeping closets

21. Mechanical, electrical and telecom

22. Service shaft

23. Interstitial space

24. Stair

Each of these is explicitly associated to a set of attributes; these along with their values have been compiled from design guidelines [3]-[5], [22]-[24]. When processed by the LVDA these attributes are embedded in the BIM database, enhancing the semantics of the BIM model to both; support LVDA proprietary computations, and other types of BPS assessment that might happen downstream the design process.

For the implementation of the LVDA two pieces of contemporary technologies have been selected; Firstly, the extensive semantics in the BIM data model; Secondly, the estimation of engineering data using normative calculations. Lee et al. [28] demonstrated how semantically rich environments found in BIM can be used for the derivation of spatial relationships in building design. Their research also improved the decision process in early stages of design, their assessment structure was based on a standalone rule-based BIM checker. Park and Augenbroe [29], [30], demonstrated the viability of using normative calculations for energy consumption estimation; they also pointed that normative calculations are well suited for sensitivity/feasibility studies for buildings.

During the initial stages of this research it was identified the need for the LVDA to provide close to real time user feedback with limited inputs, to suit the characteristics of laboratory PCD workflows. Therefore, the software was developed requiring minimal interaction from users. Another aspect identified early in the development of the LVDA was the necessity for it not to disrupt the flow of the design process. For this reason, instead of developing a standalone application, all the modules of the LVDA have been embedded in a CAD BIM in the form of plug-in, in this case we have selected Autodesk Revit as the host.

The UI designed for the LVDA is based on the concept of simplicity. There are only two buttons in the LVDA UI, they are constructed in the Revit Ribbon panel. Within the LVDA architecture, these two buttons represent two modules, these take on the responsibility of controlling the execution of all other functions in the LVDA. If data needs to by dynamically loaded into the system, users might be required to act such as to point the location an input file or connect the computer to the World Wide Web. The first of these modules controls the operations related to the computations of ventilation semantics related to the model, the second module, controls the operations related to evaluation the layout properties and to test the possible geometrical solution for ventilation system routing.

\subsection{Development of the Air Distribution Routing Estimator Module (ADREM)}

The ADREM verifies the layout spatial properties and generates a routing solution based on domain heuristics and the layout, it also analyses the routing and the performance of the air distribution system. These types of assessment are not commonly available in traditional 
engineering of ventilation systems in PCD. During this phase, the LVDA derives the following from the BIM; building morphology, airflow pressure structure, and spatial adjacencies. These provide the LVDA with the information required for the estimation of the properties of the air distribution systems that would better suit the PCD layout.

\subsubsection{Morphology Derivation Module (MDM)}

The first step during the execution of the ADREM is the derivation of the building layout features; this has a direct impact on the order in which the ADREM algorithms are executed. The MDM first analyses the PCD BIM looking for the indication of the design being either an interstitial or a service shaft type of facility. The MDM informs the LVDA about the behavioural constraints to be applied for the ventilation system engineering estimation. We deal with the derivation of laboratory building morphology with the application of three different approaches; firstly, checking for the existence of interstitial spaces in the BIM, this is done by querying the BIM for spaces with name: interstitial space, this is understood by the system as a dedicated space capable of servicing process driven spaces located directly above or below. Secondly searching for the spatial properties indicating the presence of process driven spaces with floor to floor height capable of hosting interstitial spaces, and thirdly for those layouts in which none of the previous indicators can be found, this is understood as a layout belonging to a service shaft or service corridor building typology.

\subsubsection{Interstitial space routing}

The existence of interstitial space objects in the BIM is interpreted by the LVDA as the intent of having reconfigurable service systems for the laboratory. It also defines the relationship between service spaces and conditioned spaces; in this case the system connectivity is constructed in the vertical plane. We propose here that such relationships can be extracted from the vertical adjacency which lies implicit in the BIM data structure. Deriving the vertical adjacencies in laboratory layouts Interstitial typologies requires form the LVDA to analyse the design vertical properties; the LVDA retrieves the boundary geometry in the serviced spaces and evaluates their relation to the boundaries of the interstitial space. It is important to note, that besides the explicit flexibility provided by interstitial typologies, the operational constraints and best practices for laboratories remain. Therefore, practices such as placing the insertion point for the HVAC close to the space occupant's entry/exit is considered a good practice. Unlike later design phases, where doors can be utilized to point to the entry/exit of the space, in PCD the location of the entry point of each space is derived by the system, and explicitly associated to the interstitial space. This is then used as target point for each of the branches of the distribution system within the interstitial space itself. Then entry point is estimated by setting it at the midpoint of the common boundary between the serviced space and the circulation space. This approach still needs for the definition of a start space for the branch, such as a location of the fan, therefore in this model end users are required to provide its location.

\subsubsection{Space based routing}

In this approach MDM verifies the nonexistence of interstitial spaces, but the existence of service corridors; this is interpreted by the LVDA as the designer's intention of having the ventilation system running through them. The MDM identifies then all the spaces that could be serviced by routing the ventilation system through the service corridor and proceeds to estimate the route. The routing functions used here are based in on the definition of a vertical drop adjacent to a service corridor space. If no vertical drop is defined, the LVDA builds the route by tagging the space requiring the most ventilation in the entire layout as start. 
The system considers this space, as suitable for the location of the system's vertical drop. When the layout contains service shafts and no service corridors, the LVDA uses the shafts as vertical drops for the ventilation system ducts and assumes that the designer's intention is to host the ventilation branch within the circulation area. The derivation of the morphology of each ventilation branch is responsibility of the routing algorithm of the Spatial Adjacency Analysis Module (SAAM).

\subsubsection{Spatial Adjacency Analyzer Module (SAAM)}

An important functionality of the LVDA is the analysis of spatial adjacencies, this allows for the routing of the ventilation system to supply spaces even if ante rooms are part of the layout, spaces such as; lobbies, sound locks or layouts including spaces Biosafety level classification (BSL), such as BSL-3 and BSL-4 can be processed. The SAAM analyses each of the branches of the graph looking for the following;

1. Target spaces for the ventilation system not directly adjacent to the service space but, that can be reached by going through a non-process driven space

2. Non-serviced spaces directly adjacent to the service space that might serve as anteroom for process driven spaces.

The SAAM adds to the space list all suitable spaces directly adjacent to the service corridor, then recursively analyses each space in the list and all the spaces adjacent to them, this is described here as second level spatial adjacency analysis (Fig. 1). The SAAM checks the second level spatial adjacency and the space classification included in the LVDA which indicates if the space must be supplied by the ventilation system, and therefore added to the list. The SAAM iterates through the space list until it runs in to; a previously visited target, a service space or a space for which there is an operational constraint for running the ventilation system through it. All the target spaces identified by the SAAM and their ventilation requirements are added into the ventilation branch properties and flagged as already included in a ventilation system.

\subsection{SAAM Implementation structure}

\subsubsection{Directional Airflow Structure Analyzer (DASA)}

The LVDA analyses the layout directional air-flow pattern, this operation is based on safety guidelines and is usually done by the engineer, it helps to identify the compliance of negative

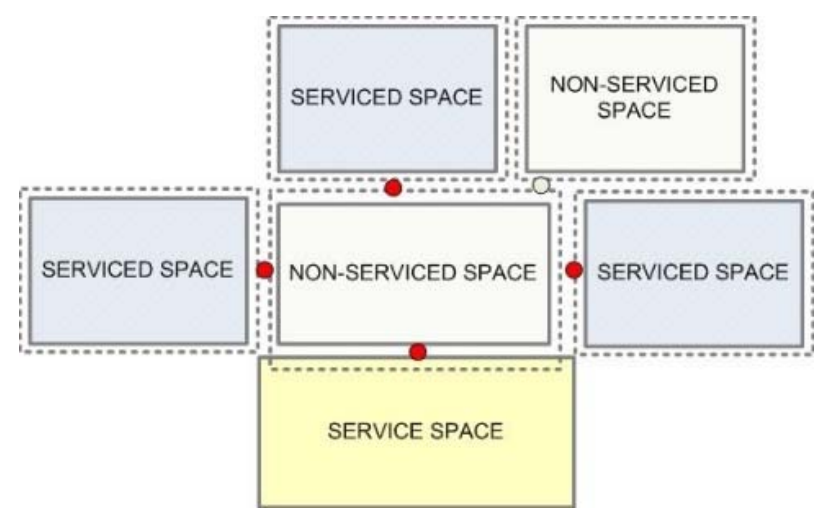

Figure 1: Second level spatial adjacency derivation. 


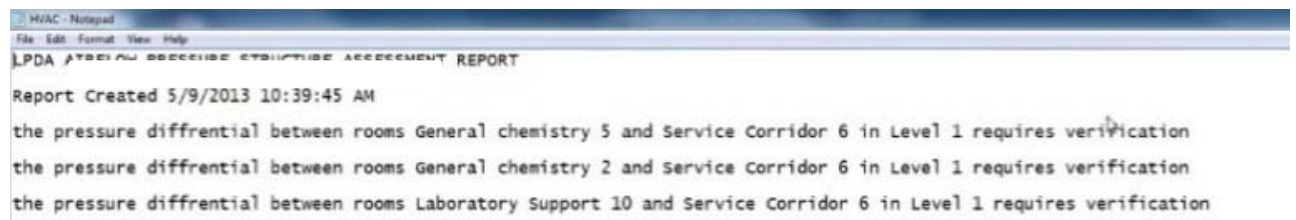

Figure 2: DASA text-based error report.

air pressure airflows towards process driven spaces. This is usually incorporated in the design after PCD. The DASA retrieves from the BIM all spaces classified by the system as process driven spaces and labels them as targets. The DASA then interrogates each target regarding it's spatial adjacencies, it checks for the directional air flow among the target and its surrounding spaces. If during the analysis an airflow pattern which might allow for air to escape the target is detected, a warning is generated by the system, in it the error space the target and the building location information are identified. After the analysis is completed, a list is saved as document (.txt) (Fig. 2) and the end user is informed about the existence of errors in the BIM and location of the error file.

\subsection{Ventilation Routing Estimator Module (VREM)}

Another major function in the LVDA is the VREM, during its execution the building morphology is evaluated to provide an estimation for the ventilation system routing. At this stage the routing of air distribution ducts is derived, duct geometry, and ventilation system attributes are estimated. The potential locations for the vertical drops is defined and informed to the end user through Revit's UI.

\subsection{Routing estimation derivation}

BIM technologies have been used to automate several aspects of building design assessment, Lee et al. [28] used BIM data for a variety of assessments during PCD, among these; circulation and security validation. In their study circulation paths were analysed regarding provisions from design guidelines. In their work, Lee et al. [28] used graphs traversing all the possible circulation paths between what they call start and target spaces. An extrapolation of this approach is used in this research for the estimation of HVAC routing. We identify what spaces in the model have the required conditions to host the distribution ducts and derive the apace adjacencies between them and all the serviced spaces. We propose the representation of the service-serviced spatial adjacency structure in a Service Adjacency Graph (SAG).

The proposed SAG allows the LVDA to evaluate domain specific constraints regarding different types of ventilation system. The LVDA incorporates a space classification algorithm that allows for the construction of a domain specific graph of the layout. The space classification used here is as follows: Service spaces: spaces suitable for elements of the ventilation system running through them, these include interstitial space, service corridor, shaft, and corridor. Serviced spaces: spaces for which the LVDA defines ventilation requirements, among these, process driven spaces, and depending on design others such as offices, and ancillary spaces. Non-serviced spaces: spaces for which the LVDA defines no ventilation requirements. 


\subsection{Derivation of spatial adjacency}

The construction of the SAG is based on the VREM capability to retrieve the properties of the layout, indicating both; types of spatial adjacencies, and the estimation of the ventilation system connection points towards the serviced space. To obtain this information, several geometric operations need to be performed. Some of these such as polygon offset, and polygon intersections, are not available through Revit's API. To enable the LVDA to perform these it was necessary to link to ti an external geometric library. Many geometric libraries are available for open source use, based on; implementation requirements, language compatibility and overall processing performance, we have chosen the Clipper geometric library (http://angusj.com/delphi/clipper.php).

\subsubsection{Deriving special spatial adjacency}

The derivation of spatial adjacency enables the VREM to check the spatial relationship between the service space and potential serviced spaces. To derive the spatial adjacency the algorithm extracts the boundary information of each serviced space, and then it translates the line-based representation coming from Revit into a polygon object, proceeds to offset the polygon by a predetermined value. Then, to determine the actual adjacency the VREM places a point at the midpoint of each edge of the polygon, then the VREM uses the Point in Polygon (PIP) test to check if one of these points is placed inside the service space polygon (Fig. 3). If the test returns true, the serviced space is added to the target space set. If the test returns false, each of the edges of the will be recursively processed and tested again in a brute force approach until either finds the adjacency information or discards the space being tested. The PIP test is based on the crossing number geometric operation, in it a ray starting in the inspected point crosses the boundary of the polygon, if the number of crossings is even; the point is outside of the polygon, if the number is odd; the point is inside the polygon.

Every time an adjacency is detected by the VREM, the space being tested is added to the list of spaces needing to be serviced. The properties of the ventilation branch are evaluated regarding the ventilation requirements contained in each space set which in turn determines the maximum diameter of ducts and the required space to fit these in the service space.

\subsubsection{Route Estimation Module (REM)}

The points constructed by the VREM during the PIP test, are interpreted as connecting points for the ventilation system; they are used as targets to estimate the routing system. The estimation of the routing involves also the extraction of the geometric properties of the space

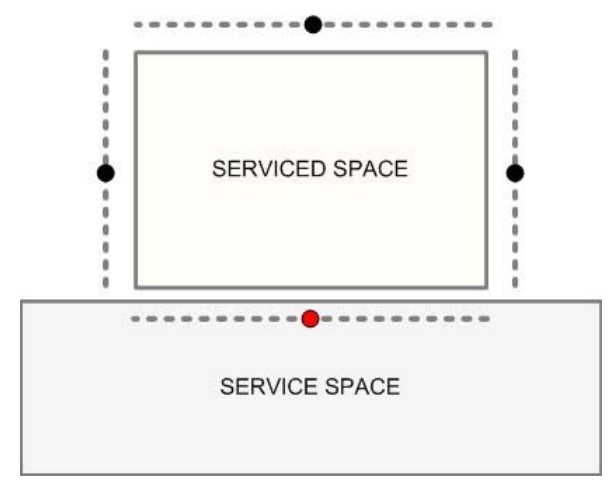

Figure 3: Adjacent space derivation. 


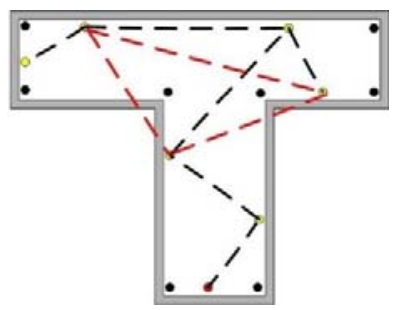

Figure 4: Routing derivation on concave spaces.

in which the ventilation branch operates and the optimization of the route in terms of system performance. For the estimation of the ventilation system routing the REM takes the vertex (V) identified as the start of the system and looks for connection to the closest unvisited $\mathrm{V}$ (target), once the closest is identified an edge is constructed by the REM, this new edged (E) is tested for possible intersection with the boundary of the service space, if intersection exists, it means the constructed $\mathrm{E}$ is out of space bounds and needs to be discarded. Then the connection is tested to the following close $\mathrm{V}$. Each time a $\mathrm{V}$ is added a new $\mathrm{E}$ is created using the new $\mathrm{V}$ and the previous $\mathrm{V}$, each $\mathrm{E}$ is tested for path self-intersection and out of service space bounds condition, if this test return true, the algorithm traces back to previous $\mathrm{V}$ and tries a new connection, the process is iterated until all unvisited $\mathrm{V}$ are added to the path. If no target space is directly visible the algorithm tests the closest $\mathrm{V}$ of the circulation space and then checks for more space target $\mathrm{V}$, this approach allows the REM to operate in both convex and concave types of service spaces (Fig. 4).

The computational implementation of the shortest path in the LVDA prototype uses a traditional computer science algorithm, developed by Robert C. Prim in 1957, Prim's algorithm solves the minimum spanning tree in computational weighted graph structures, it searches the shortest route between all the nodes in the graph. The algorithm starts from the source $\mathrm{S}$ and searches among all the adjacent nodes in the graph which have not been relaxed or visited. Calculates the distance to them, adds to the graph the closest node $\mathrm{V}$, proceeds to flag it as visited and set the $\mathrm{V}$ as $\mathrm{S}$ and continues through the graph until all nodes are visited. Unlike the original Prim's the LVDA algorithm starts from a defined node and every time a new node is added the edge created, is also tested for its relation to the service space bounds, This algorithm deals with the metric properties of the path, meaning the length of the route and the number of connecting components, both of which influence the ventilation system performance.

\section{SUMMARY}

This research evaluated the possibilities of integrating spatial properties evaluation to BIM enabled laboratory PCD, the implementation was done to support the automation of domain specific knowledge-based ventilation engineering in PCD. This was done to provide better integration of ventilation engineering on to PCD design of laboratories. There are intrinsic limitations to this research that are given by the nature of BIM CAD models for laboratory PCD, some of this can be found on the level of completeness of the BIM and how this can affect the accuracy of the test. One of the most important aspects of this research is effort in embedding spatial properties evaluation in the form of expert knowledge and computer algorithms, allowing to non-experts the evaluation of domain specific aspects of the design layout. When evaluating the types of analysis and the range of results the provided by the LVDA prototype to those of traditional processes supported by engineers and BPS, the latter 
covers a wider spectrum of analysis parameters which undeniably provides a more comprehensive understanding of the expected building behaviour and provides the evaluation of "what if" scenarios. Traditional BPS might produce more accurate results, but is important to evaluate the correlation between the types of evaluation and the speed of the feedback produced by the different approaches, and how these fit the different stages of design, in other terms what is the right tool for the right design phase Different approaches have been developed in this research to extend the scope of the assessment data of laboratories PCD, among these the capability of evaluating the spatial adjacencies of the building layout in terms of building systems engineering and routing. This type of analysis has been deemed useful for several other areas of PCD feedback and is easy to foresee a wider range of application of these on either other type of assessments or different types of building systems. In terms of the efficiency of the system developed here, at this point and without extensive end user evaluation it is hard to produce hard metrics regarding its ease of use, but it must be noted the comparative efficiency in two specific areas, firstly, in the process execution speed (compare the two developed process models), secondly the amount of data items required by both the traditional approach and by the LVDA prototype. Also it is important to mention that; at the level of efficiency metrics even for traditional approaches, it is hard to evaluate their accuracy in predicting behaviour when compared with the actual building operation, but it is important to notice that the estimation of systems proposed here is not meant to simulate the behaviour of the building or the system associated to it, but to provide a rigorous approach for the estimation of ventilation system engineering to be used for design decision making, either for the purpose of design modifications evaluation or for best alternative selection.

\section{REFERENCES}

[1] Eastman, C.M., BIM Handbook: A Guide to Building Information Modeling for Owners, Managers, Designers, Engineers and Contractors, Wiley, 2011.

[2] Bazjanac, V., Acquisition of Building Geometry in the Simulation of Energy Performance, Citeseer, 2001.

[3] McIntosh, I.B.D., Dorgan, C.B. \& Dorgan, C.E., ASHRAE Laboratory Design Guide, American Society of Heating, Refrigerating and Air-Conditioning Engineering, 2001

[4] Health, O.o.R.F.N.I.o., NIH Design Policies and Guidelines, Department of Health and Human Services USA, 2003.

[5] Facilities, T.N.I.o.H.D.o.T.R.O.o.R., NIH Design Requirements Manual, Biomedical Laboratories \& Animal Research Facilities, The National Institutes of Health, USA, 2008.

[6] Bennett, A.M., Parks, S.R. \& Benbough, J.E., Development of particle tracer techniques to measure the effectiveness of high containment laboratories. Journal of the American Biological Safety Association, 10(3), p. 139, 2005.

[7] General Services Administration GSA, Facilities Standards for the Public Buildings Service (P-100), Washington, DC, 2010.

[8] BIM FORUM, Level of Development (LOD) Specification, Building Smart International, Online. https://bimforum.org/lod/.

[9] Sheward, H. \& Eastman, C., Preliminary Concept Design (PCD) Tools for Laboratory Buildings, Automated Design Optimization and Assessment Embedded in Building Information Modeling (BIM) Tools, Computer Aided Architectural Design Futures, Belgium, 2011.

[10] Hegazy, T., Zaneldin, E. \& Grierson, D., Improving design coordination for building projects. I: Information model. Journal of Construction Engineering and Management, 127(4), pp. 322-329, 2001. 
[11] Mokhtar, A., Bedard, C. \& Fazio, P., Information model for managing design changes in a collaborative environment. Journal of Computing in Civil Engineering, 12(2), pp. 82-92, 1998.

[12] Welle, B., Haymaker, J., Fischer, M. \& Bazjanac, V., CAD-Centric attribution methodology for multidisciplinary optimization environments: Enabling parametric attribution for efficient design space Formulation and Evaluation. Journal of Computing in Civil Engineering, 28(2), pp. 284-296, 2014.

[13] Holzer, D., Sense-making across collaborating disciplines in the early stages of architectural design, RMIT University, 2009.

[14] Chaszar, A., Blurring the Lines, Academy Press, 2006.

[15] Trcka, M. \& Hensen, J., Overview of HVAC system simulation. Automation in Construction, 19(2), pp. 93-99, 2010.

[16] Rousseau P.G. \& Mathews, E.H., Needs and trends in integrated building and HVAC thermal design tools. Building and Environment, 28(4), pp. 439-452, 1993.

[17] Augenbroe, G., Building simulation trends going into the new millennium. Building Simulation, 7, 2001.

[18] Park, C.S., A Building Performance Toolkit for GSA, Interim Report, College of Architecture, Georgia Institute of Technology, Atlanta, GA, 2002.

[19] Bazjanac, V., Maile, T., O’Donnell, J., Rose, C. \& Mrazovic, N., Data environments and processing in SEM-automated simulation with EnergyPlus CIB. 28th International Conference, CIB, France, 2011.

[20] Sanguinetti, P., Abdelmohsen, S., Lee, J., Lee, J., Sheward, H. \& Eastman, C., General system architecture for BIM: An integrated approach for design and analysis. Advanced Engineering Informatics, 26(2), pp. 317-333, 2012.

[21] Handbook-Fundamentals, A., American Society of Heating, Refrigerating and AirConditioning Engineers, Inc., 2013.

[22] Chosewood, L.C. \& Wilson, D.E., Biosafety in Microbiological and Biomedical Laboratories, DIANE Publishing, 2007.

[23] Dahan, F., Laboratories: A Guide to Master Planning, Programming, Procurement, and Design, Norton, 2000.

[24] DiBerardinis, L.J., Baum, J.S., First, M.W., Gatwood, G.T. \& Seth, A.K., Guidelines for Laboratory Design: Health, Safety and Environmental Considerations, John Wiley \& Sons, 2013.

[25] Bell, A.A., HVAC Equations, Data, and Rules of Thumb, McGraw-Hill, 2000.

[26] Todesco, G., Integrated designs and HVAC equipment sizing. ASHRAE Journal, 46, pp. 42-47, 2004.

[27] Kimura, F., Ariyoshi, H., Ishikawa, H., Naruko, Y. \& Yamato, H., Capturing expert knowledge for supporting design and manufacturing of injection moulds. CIRP Annals-Manufacturing Technology, 53(1), pp. 147-150, 2004.

[28] Lee, J., Eastman, C.M., Kannala, M. \& Jeong, Y., Computing walking distances within buildings using the universal circulation network. Environment and Planning B: Planning and Design, 37(4), pp. 628-645, 2010.

[29] Park, C.S. \& Augenbroe, G., A Building Performance Toolkit for GSA College of Architecture, Georgia Institute of Technology, Atlanta, GA., 2004.

[30] Park, C.S. \& Augenbroe, G., Benchmarking of a Building Performance Assessment Toolkit ASCE, 2004. 